\title{
Structure et caractéristiques de la flore adventice de la ferme agricole de l'Université Gaston Berger de Saint-Louis (Sénégal)
}

\author{
Bassene César \\ Mansaly Nicolas
}

UFR des Sciences Agronomiques, de l'Aquaculture et des Technologies Alimentaires, Université Gaston Berger de Saint Louis, Saint Louis, Sénégal

Mballo Rahimi

Camara Abdoul Aziz

Mbaye Mame Samba

Noba Kandioura

Laboratoire de Botanique et Biodiversité, Département de Biologie Végétale,

Faculté des Sciences et Techniques, Université Cheikh Anta DIOP,

Dakar-Fann, Sénégal

Doi: 10.19044/esj.2018.v14n24p229 URL:http://dx.doi.org/10.19044/esj.2018.v14n24p229

\begin{abstract}
This present study was conducted to determine the structure and characteristics of the adventitious flora of the agricultural farm of Gaston Berger University (UGB) located in Saint Louis, Senegal. A species inventory was carried out using the "tour de champ" technique which consists of going through a plot in different directions and recording all the species present in the studied area. The inventoried flora is composed of 149 species distributed among 90 genera belonging to 30 families which are dominated by the Poaceae (18.8\%) and the Fabaceae (15.4\%). This flora is also characterized by the predominance of the therophytes and the chamephytes which represent $79.9 \%$ and $10.7 \%$ of recorded species, respectively. From a chorologic standpoint, pantropical (29.5\%) and African (28.2\%) species account for more than half of the species $(57.7 \%)$. The quantitative analysis of this flora reveals that rare or accidental species are more represented (52\%) followed by frequent species (16\%) and secondary species (15\%).
\end{abstract}

Keywords: Flora, adventitious, structure, characteristics, agricultural farm, North of Senegal 


\section{Résumé}

Cette étude porte sur la flore adventice dans la ferme agricole de 1'Université Gaston Berger (UGB) de Saint Louis. Elle a vise à déterminer la structure et les caractéristiques de la flore adventice de cette ferme. Elle est réalisée par la méthode de relevés phytosociologiques. L’inventaire a été effectué suivant la technique du « tour de champ » qui consiste à parcourir une parcelle dans différentes directions et inventorier toutes les espèces levées sur la surface d'observation. La flore inventoriée est composée de 149 espèces réparties entre 90 genres appartenant à 30 familles. Les Poaceae et les Fabaceae sont les familles dominantes avec respectivement une contribution de $18,8 \%$ et $15,4 \%$ de la totalité des espèces recencées. Cette flore est caractérisée par la prédominance des thérophytes avec 79,9\% suivi des chaméphytes avec $10,7 \%$. Sur le plan chorologique, elle est composée essentiellement d'espèces pantropicales $(29,5 \%)$ et africaines $(28,2 \%)$ qui présentent plus de la moitié des espèces $(57,7 \%)$. L'analyse quantitative de cette flore révèle que les espèces rares ou accidentelles sont plus représentées $(52 \%)$ suivies des espèces fréquentes $(16 \%)$ et des espèces accessoires $(15 \%)$.

Mots clés : Flore adventice, structure, caractéristiques, ferme agricole, Nord du Sénégal

\section{Introduction}

Au Sénégal l'agriculture constitue la principale source de nourriture, de revenus et d'emploi pour les populations rurales (Noba et al., 2002). Elle occupe près de $70 \%$ de la population (Noba, et al., 2004 ; Mbaye, 2013) et repose, à la fois, sur des cultures de rente (arachide, coton), sur des cultures vivrières de subsistance (mil, sorgho, maïs) et sur la culture du riz (ISRA, 2008). Ce secteur agricole connait de nombreux problèmes dus aux facteurs biotiques et abiotiques (Bassène et al., 2014). Parmi les facteurs biotiques les adventices constituent une contrainte majeure à la production des cultures et entrainent de ce fait une baisse considérable de leur rendement (Noba, 2002 ; Bassène et al., 2012 ; Mbaye, 2013). Comme dans toute exploitation agricole, l'émergence des adventices est inévitable. La pression de ces adventices enttraîne une perte énorme de temps pour les contrôler et aussi une baisse de la production causée par leur concurrence vis-à-vis de l'eau, des éléments minéraux et de la lumière (Bassène, 2014). Ces adventices des cultures sont responsables de $5 \%$ des pertes de récolte en zone tempérée et généralement de plus de $25 \%$ en zone tropicale (Le Bourgeois \& Marnotte, 2002). Dans ce site, la flore adventice n'a jamais fait l'objet d'étude.

Au regard de la compétitivité des adventices, la présente étude sur la flore adventice est entreprise afin d'identifier les informations utiles sur une 
flore adventice permettant de proposer une méthode efficace de gestion de ces adventices. Elle consiste à inventorier les adventices et à déterminer la structure et les caractéristiques de cette flore. Ainsi, les spectres taxonomique, biologique, chorologique et la fréquence des adventices sont investigués.

\section{Matériel et méthodes:}

\section{Site de l'étude:}

L'étude a été réalisée en 2016 et 2017 dans la ferme agricole de 1'Université Gaston Berger de Saint Louis. Cette ferme s'étend sur 30 hectares et se trouve dans l'enceinte de ladite université (Figure 1), située entre $16^{\circ} 13^{\text {' }}$ Nord et $16^{\circ} 18^{\prime}$ Ouest à, dans la région de Saint Louis au Nord du Sénégal, précisément dans le delta du fleuve Sénégal (Diack \& Loum, 2014). Dans cette ferme, les parcelles sont découpées en 0,5 ha pour des raisons d'exploitation. L'exploitation de ces parcelles n'est pas homogène. En effet, certaines parcelles sont exploitées tandis que d'autres sont laissées en jachère durant 2 ans en moyenne. Le système d'irrigation utilisé pour l'exploitation de ces parcelles est le goutte-à-goutte. L'eau utilisée pour l'irrigation des parcelles provient du bras du fleuve Sénégal appelé Djeuss, situé à $1031 \mathrm{~m}$ de la station de pompage où sont immergées deux pompes électriques (Diack, 2011).

Le climat est du type soudano-sahélien, marqué par deux saisons : une saison sèche plus longue (novembre - juin) et une saison des pluies plus courte (juillet - octobre). La moyenne annuelle de la pluviométrie est $450 \mathrm{~mm}$ environ. Cette faible pluviométrie installe un climat de type sahélien. Cependant le delta du fleuve Sénégal où se situe notre zone d'étude est soumise à une forte influence maritime qui y installe un climat sub-canarien (Diack \& Loum, 2014).

Le relief de la ferme est globalement plat. Les sols sont sableux à 1'horizon 0-50 cm et à sablo-argileuses à 50-140 cm (Diack, 2011 ; Diack \& Loum, 2014). La texture confère à ces terres une aptitude réelle à des activités agricoles et particulièrement au maraîchage et à l'arboriculture. Quelques inclusions de texture fine (argileuse) au niveau des parties dépressionnaires propices à la pisciculture sont observées (Diack, 2011). Ces zones de dépression sont inondées en saison des pluies et l'évaporation de ces eaux de pluies dans ces parties conduit à des dépôts superficiels de sel. 


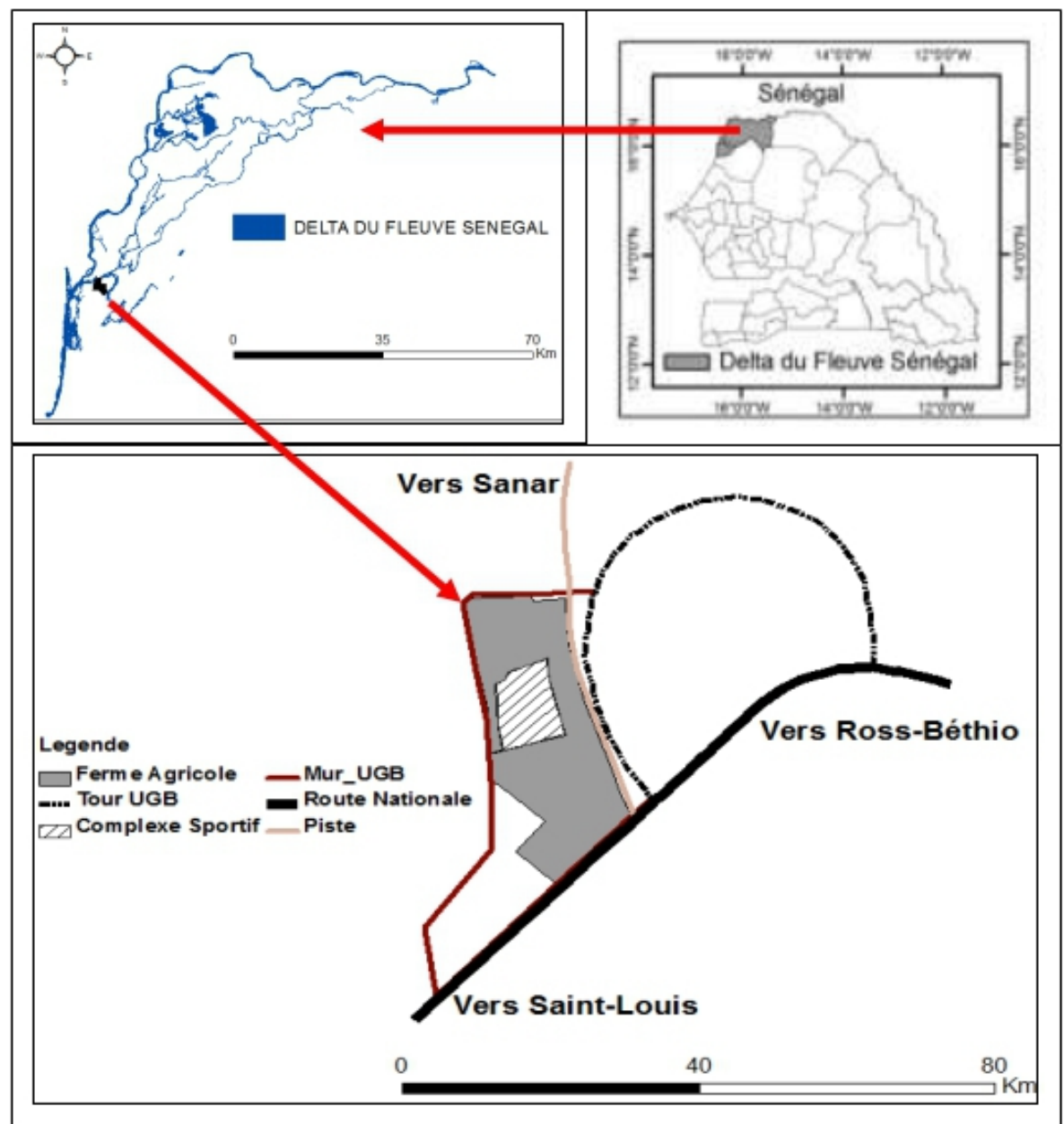

Figure 1 : Localisation de la zone d'étude (Diack \& Loum, 2014)

\section{Méthodes:}

\section{Réalisation des relevés:}

Des inventaires de la flore adventice ont été réalisés sur la ferme agricole de l'Université Gaston Berger en 2017 en saison sèche pendant la contre saisonau entre novembre et juin et au cours de la saison des pluies de entre juillet et octobre. Cette collecte de données floristiques a été effectuée sur une surface totale de 30 ha. L'aire retenue dans l'exécution des relevés est de 0,5 ha qui est en rapport avec les parcelles d'exploitation soit 116 relevés, soit 58 relevés par saison. L'inventaire est réalisé suivant la méthode de relevés phytosociologiques. La technique du «tour de champ », consistant à parcourir une parcelle dans différentes directions et à inventorier toutes les espèces émergées; a été utilisée (Noba, 2002). L'identification des espèces a été réalisée sur place et certaines plantes n'ont pas pu être déterminées immédiatement. Un code provisoire a été attribué à ces plantes pour une 
identification ultérieure. La détermination de ces espèces a été effectuée à l'aide des flores notamment la flore illustrée du Sénégal (Berhaut, 1971-1991), la flore du Sénégal (Berhaut, 1967), la nouvelle flore illustrée (Mougnier, 2008); la flore Adventrop (Le Bourgeois \& Merlier, 1995), la flore de Merlier \& Montegut (1982); la flore de Akobundu \& Agyakwa (1989) et l'Herbier du Département de Biologie Végétale de la Faculté des Sciences et Techniques de 1'Université Cheikh Anta Diop de Dakar.

La nomenclature utilisée est celle de Lebrun \& Stork (1991, 1992, 1995 et 1997) et prend en considération la classification d'Angiosperms Phyllogeny Group (APG III, 2009).

La classification de (Raunkier, 1934) adaptée à la zone tropicale où la saison défavorable correspond à la saison sèche (Trochain, 1966) a été utilisée pour distinguer les types biologiques.

En ce qui concerne la répartition géographique, les critères utilisés sont ceux de Bassène et al. (2012 et 2014), Sarr et al. (2007), Noba et al. (2004), Poilecot (1995 et 1999), et de Traoré \& Maillet (1992) et de la base de données du jardin botanique de la ville de Genève.

La fréquence relative $(\mathrm{Fr})$ de chaque espèce est déterminée à partir du rapport entre le nombre de fois que cette espèce est rencontrée et le nombre total de relevés réalisés multiplié par 100.

$\mathrm{Du}$ point de vue quantitatif, les espèces sont classées selon leur fréquence relative en rapport avec leur équivalence suivant l'index de Caratini (1985) (Tableau 1).

Tableau 1: Index de (Caratini, 1985)

\begin{tabular}{|c|c|c|}
\hline Fréquences & Index & Qualification \\
\hline 0,8 à 1 & V & Constante \\
\hline 0,6 à 0,8 & IV & Abondante \\
\hline 0,4 à 0,6 & III & Fréquente \\
\hline 0,2 à 0,4 & II & Accessoire \\
\hline 0 à 0,2 & I & Rare ou Accidentelle \\
\hline
\end{tabular}

\section{Résultats:}

\section{Structure de la flore:}

Sur la base de 116 relevés réalisés dans les parcelles d'exploitation de la ferme agricole de 1'Université Gaston Berger, 149 espèces ont été recensées. Ces 149 espèces sont réparties dans 90 genres appartenant à 30 familles (Tableau 2).

Tableau 2: Liste des espèces recensées avec leur Répartition Phytogéographiques (RPG), Types Biologiques (TB), Fréquences relatives (Fr) et leurs indices (Ind.)

\begin{tabular}{|c|l|c|c|c|c|}
\hline \multicolumn{1}{|c|}{ Familles } & \multicolumn{1}{|c|}{ Espèces } & RPG & TB & Fr & Ind \\
\hline \multirow{3}{*}{ Aizoaceae (D) } & Sesuvium hydaspicum (Edg.) Gonc. & Cosm & Th & 21,87 & II \\
\cline { 2 - 6 } & Sesuvium portulacastrum L. & Amas & Ch & 3,12 & I \\
\cline { 2 - 6 } & Trianthema pentandra L. & Masu & Th & 18,75 & I \\
\hline
\end{tabular}




\begin{tabular}{|c|c|c|c|c|c|}
\hline & Trianthema portulacastrum L. & Masu & Th & 46,87 & III \\
\hline \multirow{5}{*}{ Amaranthaceae (D) } & Achyranthes aspera L. & Cosm & Th & 25,00 & II \\
\hline & Aerva javanica (Burm.) Juss. & Af & Th & 37,50 & II \\
\hline & Amaranthus graecizans L. & Cosm & Th & 28,12 & II \\
\hline & Amaranthus spinosus L. & $\mathrm{Pt}$ & Th & 9,37 & I \\
\hline & Amaranthus viridis $\mathrm{L}$. & $\mathrm{Pt}$ & Th & 3,12 & I \\
\hline Amaryllidaceae (M) & Pancratium trianthum Herb. & Af & $\mathrm{Ge}$ & 78,12 & IV \\
\hline Araceae $(\mathrm{M})$ & Stylochiton lancifolius Kots. Et Peyr. & Af & $\mathrm{Ge}$ & 25,00 & II \\
\hline Apocynaceae-Asclepioideae (D) & Pergularia deamea (Forssk.) Chiov. & Af & $\mathrm{He}$ & 3,12 & I \\
\hline \multirow{9}{*}{ Asteraceae (D) } & Acanthospermum hispidum DC. & $\mathrm{Pt}$ & Th & 3,12 & I \\
\hline & Blumea mollis (D. Don.) Merrill. & $\mathrm{Pt}$ & Th & 9,37 & I \\
\hline & Blumea aurita (L.) DC. & Cosm & Th & 15,62 & $\mathrm{I}$ \\
\hline & Coniza aegyptica (L.) Ait. & $\mathrm{Pt}$ & Th & 6,25 & I \\
\hline & Eclipta alba (L.) Hassk. & $\mathrm{Pt}$ & Th & 3,12 & I \\
\hline & Emilia sonchifolia (L.) DC & $\mathrm{Pt}$ & Th & 50,00 & III \\
\hline & Launaea intybacea (Jacq.) Beauverd. & $\mathrm{Pt}$ & Th & 93,75 & $\mathrm{~V}$ \\
\hline & Launaea taraxaxifolia (Will.) Schum. & $\mathrm{Pt}$ & Th & 15,62 & $\mathrm{II}$ \\
\hline & Tridax procumbens $\mathrm{L}$. & As & Th & 12,50 & I \\
\hline \multirow[t]{3}{*}{ Boraginaceae (D) } & Heliotropium bacciferum Forsk. & $\mathrm{Pt}$ & Th & 96,87 & $\mathrm{~V}$ \\
\hline & Heliotropium ovalifolium Forsk. & Afasu & $\mathrm{Ch}$ & 3,12 & I \\
\hline & Heliotropium subulatum (Hochst.)Auct. & Mas & $\mathrm{Ch}$ & 6,25 & I \\
\hline \multirow{2}{*}{ Capparidaceae (D) } & Cleome gynandra $\mathrm{L}$. & Masu & Th & 18,75 & I \\
\hline & Cleome tenella $\mathrm{L}$. & Af & Th & 6,25 & I \\
\hline Chenopodiaceae (D) & Salsola baryosma (Schult.) Dandy. & Afas & $\mathrm{Ch}$ & 3,12 & I \\
\hline \multirow{3}{*}{ Commelinaceae (M) } & Commelina benghalensis L. & $\mathrm{Pt}$ & Th & 9,37 & I \\
\hline & Commelina forskalaei Vahl. & $\mathrm{Pt}$ & Th & 90,62 & $\mathrm{~V}$ \\
\hline & Commelina gambiae (C.B.Clar.) Brenan & Mas & Th & 3,12 & I \\
\hline \multirow{10}{*}{ Convolvulaceae (D) } & Cressa cretica $\mathrm{L}$. & $\mathrm{M}$ & Th & 3,12 & I \\
\hline & Hewittia sublobata (L.f.) O. Kuntze. & Masu & $\mathrm{Ch}$ & 34,37 & II \\
\hline & Ipomoea asarifolia (Desr.) R. et Sch. & $\mathrm{Pt}$ & $\mathrm{Ch}$ & 18,75 & I \\
\hline & Ipomoea coptica (L.) Roth. & Masu & Th & 90,62 & $\mathrm{~V}$ \\
\hline & Ipomoea eriocarpa $\mathrm{R} . \mathrm{Br}$. & Masu & Th & 25,00 & II \\
\hline & Ipomoea pes-tigridis L. & Afasu & Th & 31,25 & II \\
\hline & Ipomoea vagans Bak. & Af & Th & 3,12 & I \\
\hline & Jacquemontia tamnifolia (L.) Griseb. & $\mathrm{Pt}$ & Th & 40,62 & III \\
\hline & Merremia aegyptica (L.) Urban & $\mathrm{Pt}$ & Th & 21,87 & II \\
\hline & Merremia pinnata Hochst & Af & Th & 3,12 & I \\
\hline \multirow{4}{*}{ Cucurbitaceae (D) } & Coccinia grandis (L.) J.O. Voigt. & Afasu & Th & 31,25 & II \\
\hline & Cucumis ficifolius A. Rich. & Afas & Ch & 12,50 & I \\
\hline & Cucumis melo var. agrestis (L.) Berh. & Masu & Th & 50,00 & III \\
\hline & Momordica balsamina $\mathrm{L}$. & Masu & Th & 56,25 & III \\
\hline \multirow{12}{*}{ Cyperaceae (M) } & Bolboschoenus maritimus (L.) Palla. & Cosm & $\mathrm{Ge}$ & 6,25 & I \\
\hline & Bulbostylis hyspidula (Vahl) Haines & Af & Th & 31,25 & II \\
\hline & Cyperus articulatus L. & $\mathrm{Pt}$ & $\mathrm{He}$ & 3,12 & $\mathrm{I}$ \\
\hline & Cyperus compressus L. & Cosm & Th & 37,50 & II \\
\hline & Cyperus difformis $\mathrm{L}$. & $\mathrm{Pt}$ & Th & 3,12 & I \\
\hline & Cyperus dives Delile & Masu & Th & 3,12 & I \\
\hline & Cyperus esculentus L. & Cosm & Th & 43,75 & III \\
\hline & Cyperus haspan L. & $\mathrm{Pt}$ & $\mathrm{He}$ & 3,12 & I \\
\hline & Cyperus iria $\mathrm{L}$. & Afasam & Th & 3,12 & I \\
\hline & Cyperus rotundus $\mathrm{L}$. & $\mathrm{Pt}$ & Th & 62,50 & IV \\
\hline & Fimbristylis exilis (Kunt.) Roem \& Sch. & $\mathrm{Pt}$ & Th & 21,87 & II \\
\hline & Kylling a pumila Michaux & $\mathrm{M}$ & $\mathrm{He}$ & 6,25 & I \\
\hline
\end{tabular}




\begin{tabular}{|c|c|c|c|c|c|c|}
\hline & Maricus ligularis (L.) Urban & $\mathrm{Pt}$ & Th & 3,12 & I \\
\hline & & Mariscus squarosus (L.) C. B. Clarke & $\mathrm{Pt}$ & Th & 56,25 & III \\
\hline \multirow{2}{*}{\multicolumn{2}{|c|}{ Euphorbiaceae (D) }} & Euphorbia forskalaei S. Gay. Boiss. & Af & Th & 15,62 & $\mathrm{I}$ \\
\hline & & Euphorbia hirta L. & $\mathrm{Pt}$ & Th & 78,12 & IV \\
\hline \multirow{23}{*}{$\begin{array}{l}\text { Fabaceae } \\
\text { (D) }\end{array}$} & \multirow[t]{3}{*}{ Ceasalpi noideae } & Cassia italica (Miller) Lam. & Afas & $\mathrm{Ch}$ & 56,25 & III \\
\hline & & Cassia obtusifolia L. & Afasam & Th & 3,12 & $\mathrm{I}$ \\
\hline & & Cassia occidentalis L. & $\mathrm{Pt}$ & Th & 21,87 & II \\
\hline & \multirow{20}{*}{ Faboideae } & Alysicarpus ovalifolius (S.\&Th.) Léon & $\mathrm{Pt}$ & Th & 93,75 & $\mathrm{~V}$ \\
\hline & & Crotalaria cylindrocarpa DC. & Af & Th & 6,25 & $\mathrm{I}$ \\
\hline & & Crotalaria glaucoides Bak. & Af & Th & 12,50 & I \\
\hline & & Crotalaria podocarpa DC. & Af & Th & 78,12 & IV \\
\hline & & Crotalaria senegalensis (Pers.) Bacle. & Af & Th & 6,25 & $\mathrm{I}$ \\
\hline & & Cyamopsis senegalensis Guill. et Perr. & Af & Th & 56,25 & III \\
\hline & & Desmodium asperum (Poir.) Desv. & $\mathrm{Pt}$ & Th & 3,12 & $\mathrm{I}$ \\
\hline & & Indigofera aspera Perr. & Af & Th & 84,37 & $\mathrm{~V}$ \\
\hline & & Indigofera astragalina $\mathrm{DC}$. & Af & Th & 6,25 & $\mathrm{I}$ \\
\hline & & Indigofera colutea (Burm.) Merrill. & Af & Th & 50,00 & III \\
\hline & & Indigofera diphylla Vent. & Af & Th & 28,12 & II \\
\hline & & Indigofera hursita $\mathrm{L}$. & $\mathrm{Pt}$ & Th & 18,75 & $\mathrm{I}$ \\
\hline & & Indigofera oblongifolia Forsk. & Afas & Th & 12,50 & $\mathrm{I}$ \\
\hline & & Indigofera pilosa Poir. & Af & Th & 65,62 & IV \\
\hline & & Indigofera senegalensis Lam. & Af & Th & 12,50 & $\mathrm{I}$ \\
\hline & & Indigofera sessiflora DC. & Af & Th & 18,75 & $\mathrm{I}$ \\
\hline & & Indigofera tinctoria $\mathrm{L}$. & Masu & Th & 34,37 & II \\
\hline & & Rothia hirsuta (Guill. et Perr.) Baker. & Af & $\mathrm{Ch}$ & 9,37 & $\mathrm{I}$ \\
\hline & & Sesbania pachycarpa DC. & Af & Th & 37,50 & $\mathrm{I}$ \\
\hline & & Zornia glochidiata Reichb. & Af & Th & 12,50 & $\mathrm{I}$ \\
\hline \multicolumn{2}{|c|}{ Lamiaceae (D) } & Hyptis suaveolens Poit. & Mas & Th & 3,12 & $\mathrm{I}$ \\
\hline \multicolumn{2}{|c|}{ Liliaceae (M) } & Dipcadi tacazzeanum (H.) Baker & Af & $\mathrm{Ge}$ & 46,87 & III \\
\hline \multirow{8}{*}{$\begin{array}{l}\text { Malva ceae } \\
\text { (D) }\end{array}$} & \multirow{4}{*}{ Malvoideae } & Abutilon pannosum (Forst.) Sclecht. & Masu & Th & 15,62 & $\mathrm{I}$ \\
\hline & & Hibiscus asper Hook. & Af & Th & 6,25 & $\mathrm{I}$ \\
\hline & & Pavonia zeylanica Cav. & Afas & Th & 46,87 & III \\
\hline & & Sida rhombifolia L. & $\mathrm{Pt}$ & $\mathrm{Ch}$ & 6,25 & $\mathrm{I}$ \\
\hline & Sterculoideae & Waltheria indica $\mathrm{L}$. & $\mathrm{Pt}$ & $\mathrm{Ch}$ & 50,00 & III \\
\hline & \multirow{3}{*}{ Tilioidae } & Corchorus aestuans L. & Masu & Th & 9,37 & $\mathrm{I}$ \\
\hline & & Corchorus tridens $\mathrm{L}$. & Masu & Th & 62,50 & $\mathrm{I}$ \\
\hline & & Corchorus urticifolius Wight et Arn. & $\mathrm{Pt}$ & $\mathrm{Ch}$ & 3,12 & $\mathrm{II}$ \\
\hline \multirow{4}{*}{\multicolumn{2}{|c|}{ Molluginaceae (D) }} & Gisekia pharnaceoides (L.) Mant. & $\mathrm{Pt}$ & Th & 56,25 & III \\
\hline & & Limeum diffusum (J. Gay) Schinz. & Af & Th & 43,75 & III \\
\hline & & Mollugo cerviana (L.) Seringe & Cosm & Th & 34,37 & II \\
\hline & & Mollugo nudicaulis Lam. & As & Th & 3,12 & I \\
\hline \multirow{2}{*}{\multicolumn{2}{|c|}{ Nyctaginaceae (D) }} & Boerhavia diffusa $\mathrm{L}$. & $\mathrm{Pt}$ & Th & 75,00 & IV \\
\hline & & Boerhavia erecta $\mathrm{L}$. & $\mathrm{Pt}$ & Th & 78,12 & IV \\
\hline \multicolumn{2}{|c|}{ Passifloraceae (D) } & Passiflora foetida L. & Af & Th & 40,62 & III \\
\hline \multicolumn{2}{|c|}{ Pedaliaceae (D) } & Cerathoteca sesamoïdes Endl. & Af & Th & 56,25 & III \\
\hline \multirow{8}{*}{\multicolumn{2}{|c|}{ Phyllanthaceae (D) }} & Phyllanthus amarus Sch. Th. & Masu & Th & 71,82 & IV \\
\hline & & Phyllanthus pentandrus Sch \& Th & Masu & Th & 3,12 & I \\
\hline & & Andropogon gayanus Kunth. & Af & $\mathrm{He}$ & 9,37 & I \\
\hline & & Antephora cristata Hach..et Durand & Cosm & Th & 15,62 & I \\
\hline & & Aristida adscensionis L. & $\mathrm{Pt}$ & Th & 46,87 & III \\
\hline & & Brachiaria lata (Schumach.) Hubb. & Af & Th & 3,12 & $\mathrm{I}$ \\
\hline & & Brachiaria ramosa Stapf & Masu & Th & 50,00 & III \\
\hline & & Cenchrus biflorus Roxb. & Masu & Th & 90,62 & $\mathrm{~V}$ \\
\hline
\end{tabular}




\begin{tabular}{|c|c|c|c|c|c|}
\hline \multirow[t]{22}{*}{ Poaceae (M) } & Cenchrus setigerus Vahl. & Af & $\mathrm{He}$ & 12,50 & $\mathrm{I}$ \\
\hline & Chloris pilosa Schumach. et Thonn. & Afas & Th & 3,12 & $\mathrm{I}$ \\
\hline & Choris prieurii Kunt. & Afas & Th & 87,50 & $\mathrm{~V}$ \\
\hline & Cynodon dactylon (L.) Pers. & Cosm & $\mathrm{He}$ & 81,25 & $\mathrm{~V}$ \\
\hline & Dactyloctenium aegyptium (L.) P.Beauv. & Masu & Th & 96,87 & $\mathrm{~V}$ \\
\hline & Digitaria ciliaris (Retz.) Koel. & $\mathrm{Pt}$ & Th & 90,62 & $\mathrm{~V}$ \\
\hline & Digitaria horizontalis Willd. & Afasam & Th & 43,75 & III \\
\hline & Echinochloa colona (L.)Link. & $\mathrm{Pt}$ & $\mathrm{He}$ & 18,75 & $\mathrm{I}$ \\
\hline & Eleusine indica (L.) Gaertn. & $\mathrm{Pt}$ & $\mathrm{He}$ & 53,12 & III \\
\hline & Eragrostis cilianensis Lutati & Masu & Th & 28,12 & II \\
\hline & Eragrostis ciliaris (L.) R. Br. & $\mathrm{Pt}$ & Th & 96,87 & $\mathrm{~V}$ \\
\hline & Eragrostis tennella Raem. et Sch. & $\mathrm{Af}$ & Th & 62,50 & IV \\
\hline & Eragrostis tremula Hochst. Steud. & Masu & Th & 78,12 & IV \\
\hline & Leptothrium senegalense (Kunt.) Clay. & Afas & $\mathrm{Ch}$ & 62,50 & IV \\
\hline & Panicum laetum Kunt. & Af & Th & 15,62 & $\mathrm{I}$ \\
\hline & Pennisetum pedicellatum Trin. & Masu & Th & 18,75 & I \\
\hline & Pennisetum violaceum (Lam.) L. Rich. & Masu & Th & 3,12 & $\mathrm{I}$ \\
\hline & Phragmites vulgaris Lam. & Cosm & Th & 3,12 & $\mathrm{I}$ \\
\hline & Setaria verticillata (L.) P. Beauv. & Cosm & Th & 28,12 & II \\
\hline & Schoenefeldia gracilis Kunt. & Mas & Th & 3,12 & I \\
\hline & Sporobolus robustus Kunt & Af & $\mathrm{Ch}$ & 3,12 & I \\
\hline & Tragus berteroniamus Schult. Auct. & Mas & Th & 18,75 & I \\
\hline Polygalaceae (D) & Polygala erioptera $D C$. & Afas & Th & 18,75 & $\mathrm{I}$ \\
\hline \multirow{3}{*}{ Portulacaceae (D) } & Portulaca foliosa Ker-Gawl. & Af & Th & 3,12 & I \\
\hline & Portulaca oleracea $\mathrm{L}$. & Cosm & Th & 40,62 & III \\
\hline & Portulaca quadrifida $\mathrm{L}$. & Masu & Th & 6,25 & $\mathrm{I}$ \\
\hline \multirow{5}{*}{ Rubiaceae (D) } & Mitracarpus villosus (Sw.) DC. & Af & Th & 71,87 & IV \\
\hline & Oldenlandia corymbosa $\mathrm{L}$. & $\mathrm{Pt}$ & Th & 43,75 & III \\
\hline & Spermacoce chaetocephala DC. & Af & Th & 6,25 & $\mathrm{I}$ \\
\hline & $\begin{array}{l}\text { Spermacoce radiata (DC.) } \\
\text { Sieber.exHiern. }\end{array}$ & Af & Th & 18,75 & I \\
\hline & Spermacoce verticillata $\mathrm{L}$. & Af & Th & 43,75 & $\overline{\mathrm{III}}$ \\
\hline Scrophulariaceae (D) & Scoparia dulcis L. & $\mathrm{Pt}$ & Th & 46,87 & III \\
\hline \multirow{4}{*}{ Solanaceae } & Datura metel L. & Cosm & Th & 75,00 & IV \\
\hline & Physalis angulata $\mathrm{L}$. & $\mathrm{Pt}$ & Th & 31,25 & II \\
\hline & Physalis micrantha Link. & $\mathrm{Pt}$ & Th & 12,50 & $\mathrm{I}$ \\
\hline & Solanum nigrum L. & Cosm & Th & 3,12 & $\mathrm{I}$ \\
\hline Typhaceae (M) & Typha australis Schumach. & $\mathrm{Pt}$ & $\mathrm{Ge}$ & 3,12 & I \\
\hline \multirow{3}{*}{ Zygophyllaceae (D) } & Tribulus terrestris $\mathrm{L}$. & $\mathrm{Pt}$ & Th & 62,50 & IV \\
\hline & Zygophyllum simplex L. & Af & $\mathrm{Ch}$ & 3,12 & $\mathrm{I}$ \\
\hline & Zygophyllum waterlotii Maire. & Af & $\mathrm{Ch}$ & 6,25 & $\mathrm{I}$ \\
\hline
\end{tabular}

Type Biologique (TB) ; Répartition Géographique (RG) ; Fréquence (Fr)

Dicotylédones (D); Monocotylédones (M)

Thérophytes (T) ; Chaméphytes (C) ; Hemicryptophytes (H) ; Géophytes (G)

Pantropicale (Pt) ; Africaine (Af) ; Afro-malgache asiatique et australienne (Masu) ;

Cosmopolites (Cosm) ; Afro-asiatique (Afas) ; Afro-malgache et asiatique (Mas) ; Afro-

asiatique et américaine (Afasam) ; Afro-asiatique et australienne (Afasu) ; Afro-

malgache (M) ; Afro-américaine (Afam) 
La structure taxonomique de la flore adventice est dominée par les Dicotylédones (Figure 2). La contribution spécifique des Dicotylédones à cette flore est de 67,11\% des espèces inventoriées. La dominance des Dicotylédones est aussi observée au niveau générique avec $65,56 \%$ contre $34,44 \%$ pour les Monocotylédones. Ce même constat est observé au niveau des familles. En effet, 83,87\% des familles appartiennent aux Dicotylédones qui dominent largement cette flore contre 16,13\% des familles de Monocotylédones.

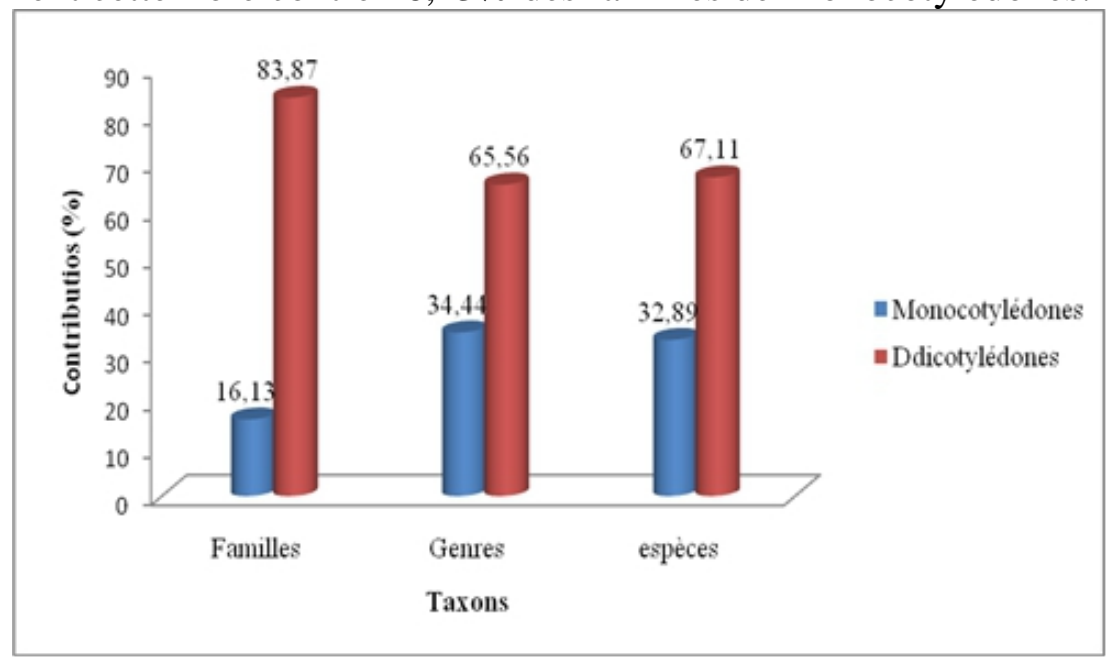

Figure 2 : Spectre taxonomique de la flore adventice inventoriée sur la ferme agricole de l’Université Gaston Berger en 2017

La répartition des espèces par famille rencontrées montre que six familles sont dominantes (Tableau 3). Il s'agit des Poaceae (18,8\%), Fabaceae $(15,4 \%)$, Cyperaceae $(9,4 \%)$, Convolvulaceae $(6,7 \%)$, Asteraceae $(6,1 \%)$ et Malvaceae avec 5,3\%. Ces familles représentent $61,7 \%$ de la totalité des familles des espèces recensées dans cette ferme agricole. Parmi ces familles, les Poaceae et les Fabaceae contribuent à elles seules 34,2\% des espèces de cette flore contre $38,2 \%$ des espèces réparties entre 24 autres familles de la flore. Il ressort de ces résultats que 11 familles sont représentées par une seule espèce. Il s'agit de 4 Monocotylédones (Amaryllidaceae, Araceae, Liliaceae et Typhaceae) et de 7 Dicotylédones (Apocynaceae-Asclepioidae, Chenopodiaceae, Lamiaceae, Passifloraceae, Pedaliaceae, Polygalaceae, Scrophulariaceae).

Tableau 3 : Liste des familles botanique et leur contribution à la flore adventice inventoriée sur la ferme agricole de l'Université Gaston Berger en 2017

\begin{tabular}{|l|c|c|}
\hline \multicolumn{1}{|c|}{ Familles } & $\begin{array}{c}\text { Nombre } \\
\text { d'espèces }\end{array}$ & $\begin{array}{c}\text { Preoportion } \\
\mathbf{\%}\end{array}$ \\
\hline Poaceae (M) & 28 & 18,8 \\
\hline Fabaceae (Caesalpinoidae et Faboidae) (D) & 23 & 15,4 \\
\hline Cyperaceae (M) & 14 & 9,4 \\
\hline Convolvulaceae (D) & 10 & 6,7 \\
\hline
\end{tabular}




\begin{tabular}{|c|c|c|}
\hline Asteraceae (D) & 9 & 6,1 \\
\hline Malvaceae (Malvoidae, Sterculoidae, Tilioidae) (D) & 8 & 5,3 \\
\hline Amaranthaceae (D) & 5 & 3,3 \\
\hline Rubiaceae (D) & 5 & 3,3 \\
\hline Aizoaceae (D) & 4 & 2,7 \\
\hline Cucurbitaceae (D) & 4 & 2,7 \\
\hline Molluginaceae (D) & 4 & 2,7 \\
\hline Solanaceae (D) & 4 & 2,7 \\
\hline Boraginaceae (D) & 3 & 2,1 \\
\hline Commelinaceae $(\mathrm{M})$ & 3 & 2,1 \\
\hline Portulacaceae (D) & 3 & 2,1 \\
\hline Zygophyllaceae (D) & 3 & 2,1 \\
\hline Capparidaceae (D) & 2 & 1,3 \\
\hline Euphorbiaceae (D) & 2 & 1,3 \\
\hline Nyctaginaceae (D) & 2 & 1,3 \\
\hline Phyllanthaceae (D) & 2 & 1,3 \\
\hline Amaryllidaceae (M) & 1 & 0,7 \\
\hline Araceae $(\mathrm{M})$ & 1 & 0,7 \\
\hline Chenopodiaceae (D) & 1 & 0,7 \\
\hline Lamiaceae (D) & 1 & 0,7 \\
\hline Liliaceae (M) & 1 & 0,7 \\
\hline Apocynaceae - Asclepioidae (D) & 1 & 0,7 \\
\hline Passifloraceae (D) & 1 & 0,7 \\
\hline Pedaliaceae (D) & 1 & 0,7 \\
\hline Polygalaceae (D) & 1 & 0,7 \\
\hline Scrophulariaceae (D) & 1 & 0,7 \\
\hline Typhaceae (M) & 1 & 0,7 \\
\hline TOTAL & 149 & 100 \\
\hline
\end{tabular}

\section{Spectre biologique:}

Les résultats sur le spectre biologique montrent que les types biologiques sont représentés par 4 catégories (Figure 3). L'analyse quantitative indique que les thérophytes (80\%) sont les types biologiques les plus dominents de la flore. Les autres catégories sont faiblement représentées avec $20,13 \%$ dont $11 \%$ des espèces sont des chaméphytes, $6 \%$ sont des hémicryptophytes et $3 \%$ sont des géophytes. En somme, la flore de cette ferme agricole est majoritairement constituée d'espèces annuelles constituées par des thérophytes (Figure 3). 


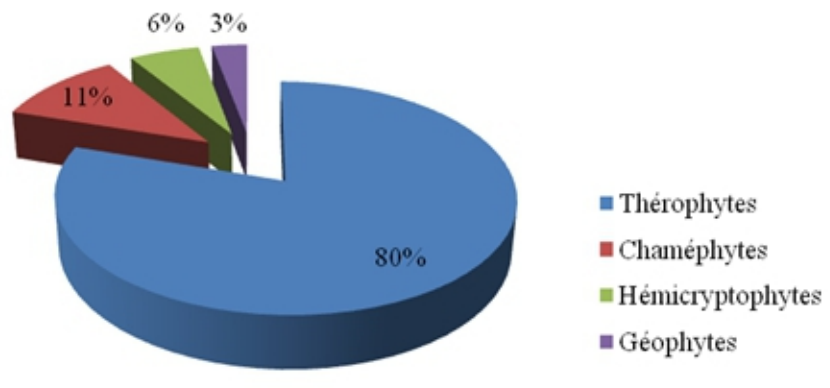

Types biologiques

Figure 3 : Spectre biologique de la flore adventice inventoriée sur la ferme agricole de l’Université Gaston Berger en 2017

\section{Spectre chorologique:}

Les adventices de la ferme présentent une assez large répartition phytogéographique (Figure 4). Les espèces d'affinités pantropicales (Pt) dominent avec $(29,53 \%)$ suivies des espèces africaines (Af) $(28,19 \%)$ et des espèces Afro-malgaches asiatiques et australiennes (Masu) $(15,44 \%)$ qui représentent au total $73,16 \%$, soit les $4 / 5$ des espèces de cette flore. Le $1 / 5$ des espèces est composé de Cosmopolites (Cosm); Afro-asiatiques; Afromalgaches et asiatiques (Mas); Afro-asiatiques et américaines (Afasam); Afroasiatiques et australiennes (Afasu); Afro-malgaches (M); Afro-américaines (Afam) $)^{\circ}$ qui représentent $23,84 \%$.

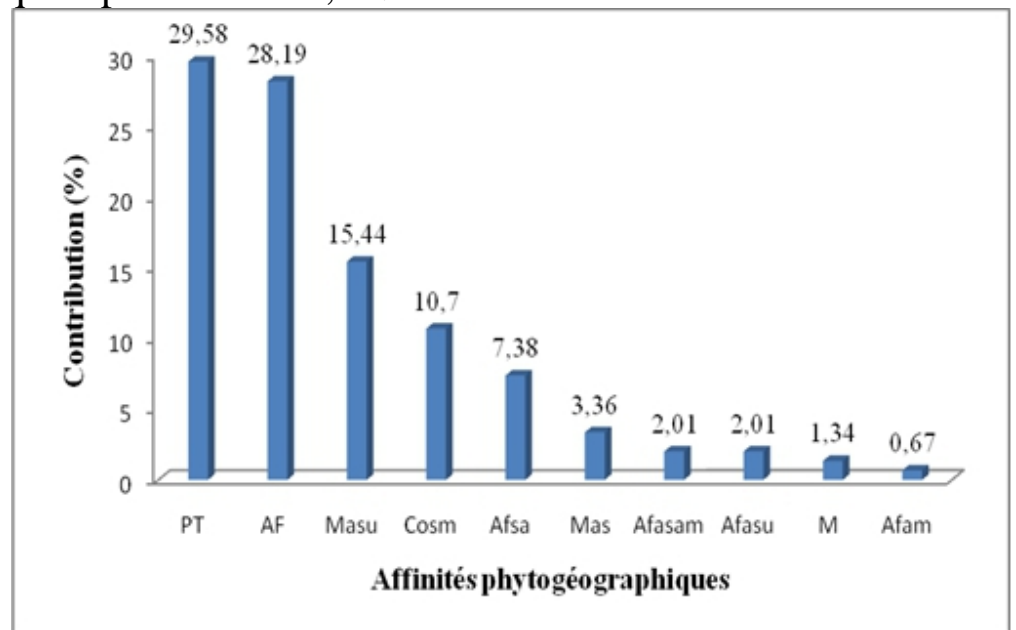

Figure 4 : Répartition phytogéographique de la flore adventice inventoriée sur la ferme agricole de l'Université Gaston Berger en 2017

Pantropicale (Pt) ; Africaine (Af) ; Afro-malgache asiatique et australienne (Masu) ;

Cosmopolites (Cosm) ; Afro-asiatique (Afas) ; Afro-malgache et asiatique (Mas) ; Afroasiatique et américaine (Afasam); Afro-asiatique et australienne (Afasu) ; Afro-malgache (M) ; Afro-américaine (Afam). 


\section{Analyse quantitative de la flore:}

L'analyse quantitative de la flore inventoriée montre que les espèces rares sont plus représentées (52\%) suivies des espèces fréquentes avec $16 \%$, des espèces accessoires avec $15 \%$, des espèces abondantes avec $9 \%$ et espèces constantes avec 8\% (Figure 5). Les espèces rares, accessoires et fréquentes représentent $83 \%$ de la totalité des espèces recencées. Ainsi, la flore de la ferme agricole est nettement dominée par les espèces rares.

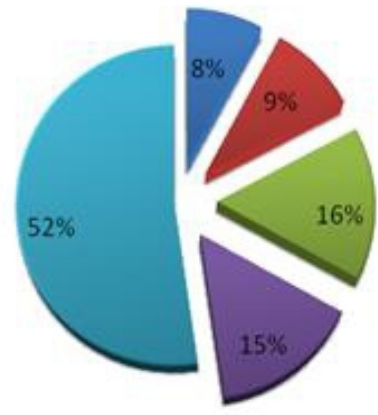

Q Constante

日abondante

u Fréquente

日accessoire

$\square$ Rare ou Accidentelle

Figure 5 : Fréquence des espèces adventices recencées sur la ferme agricole de l'Université Gaston Berger en 2017

\section{Discussion:}

L'inventaire réalisé sur la ferme agricole de l'université Gaston Berger montre que la flore adventice est composée d'environ 149 espèces réparties dans 90 genres appartenant à 30 familles. Cette flore adventice est plus diversifiée que la flore adventice des cultures d'oignons dans la zone périurbaine de Dakar (Niayes) (Sarr et al., 2007) et celle de la flore adventice du maïs dans le Sud du Bassin arachidier (Bassène et al., 2012) qui compte respectivement 131 et 128 espèces réunies dans 88 et 65 genres regroupées dans 32 et 25 familles. Elle est par contre plus pauvre que la flore des systèmes agropastoraux de la Basse Casamance (Bassène et al., 2014) qui regroupe 158 espèces réparties dans 91 genres et 37 familles.

L'analyse de la flore indique que les dicotylédones présentant environ $84 \%$ sont les plus dominantes. Ces résultats sont comparables à ceux de Zidane et al. (2010) qui ont réalisé l'étude sur les groupements d'adventices dans le Maroc occidental avec des proportions de $88 \%$ pour les dicotylédones contre $12 \%$.de monocotylédones. L'importance des familles qui occupent les 6 première positions par ordre du classement (Poaceae, Fabaceae, Cyperaceae, Convolvulaceae, Asteraceae et Malvaceae) s'expliquerait par leur aire de répartition sahélienne et par leur aptitude à s'adapter aux biotopes perturbés par les activités agricoles (Taleb et al., 1998 ; Noba, 2002 ; Bassène, 2012). A 
titre de comparaison, la prédominance des trois familles de la flore de la ferme de l'UGB est relativement identique à celle de la flore du Bassin arachidier (Noba et al., 2004) et des Niayes (Sarr et al., 2007) présentant respectivement pour Poaceae $(18,79 \%$ contre $20 \%$ et $23 \%)$, Fabaceae $(15,4 \%$ contre $8,8 \%$ et $11,5 \%)$ et pour les Cyperaceae $(9,4 \%$ contre $6,4 \%$ et $6 \%$ ). La place occupée par la famille des Fabaceae par rapport aux Cyperaceae s'expliquerait, en partie, par l'utilisation de la nouvelle classification (APGIII, 2009) qui permet de regrouper dans la famille des Fabaceae, les familles de la superfamille des Légumineuses de la classification classique (Caesalpiniaceae, Fabaceae et Mimosaceae). Ces observations rejoignent celles de Bassène et al. (2014) qui ont été notées sur la flore des systèmes agropastoraux de la Basse Casamance. Parmi ces familles dominantes, les Poaeae et les Cyperaceae appartenant à la classe des Monocotylédones représentent 28,2 \%, soit plus d'un quart des espèces inventoriées. L'importance de la famille des Cyperaceae dans la ferme pourrait s'expliquer par le fait qu'elles sont très difficiles à contrôler dans les exploitations agricoles du fait de leur mode de multiplication végétative soit par des tubercules soit par des bulbes (Bassène et al., 2012). Quant aux Légumineuses avec leur capacité de fixation de l'azote atmosphérique par une association symbiotique avec des bactéries du sol, elles pourraient être laissées volontairement dans le cadre d'adoption d'une pratiques agroécologique pour améliorer la fertilité des sols (Drevon, 2003; Schneider et al, 2015). Il est important de noter aussi que les espèces Typha australis et Phragmithes vulgaris que l'on retrouve en zone humide, sont bservées dans les parties dépressionnaires de la ferme où l'eau est souvent stagnante.

Pour les types biologiques, les résultats montrent que quatre types biologiques sont notés sur l'ensemble des espèces recensées. Les formes biologiques sont dominées par les thérophytes qui sont des espèces annuelles. En effet, la dominance des espèces appartenant à ce type biologique s'expliquerait par la productivité élevée des semences, et la phénologie parfaitement adaptée aux cultures (Hannachi, 2010). Certaines d'entre elles bouclent leur cycle en une courte durée, ce qui leur confère un réel avantage dans l'adaptation en milieu très perturbé que constitue les exploitations agricoles (Noba, 2002). Ainsi, les activités culturales laissent peu de chance au développement des espèces vivaces au profit des espèces annuelles (Bassène et al, 2012). Parmi ces activité culturales, on peut citer le travail répété du sol qui tend à éliminer les espèces pérennes (Bassène, 2014).

A la lumière de l'étude de la répartition spatiale des adventices dans le monde, nous notons la dominance par ordre d'importance des espèces à affinités pantropicales et africaines. Ces espèces seraient les mieux adaptées à la fois aux conditions climatiques et à l'écologie particulière des exploitations agricoles (Noba, 2002). Les autres espèces sont réparties dans 8 zones phytogéographiques. La faible proportion de répartition géographique de 
certaines espèces est probablement due à leur introduction accidentelle dans la ferme ou à leur non adaptabilité aux conditions pédoclimatiques de la ferme ce qui entrainerait à leur disparition (Bassène, 2014).

Les résultats de l'analyse quantitative des espèces recensées, indiquent un peuplement assez particulier des adventices dans la ferme, avec près de $60 \%$ des espèces qui sont rares ou accidentelles. Selon Rameau et al., (2008), c'est un peuplement qui n'a pas encore atteint son équilibre dynamique du fait des activités agricoles qui ont pour conséquence, la destruction d'espèces déjà établies. Parmi ces pratiques, nous notons le sarclo-binage qui favorise la remonté des semences des adventices enfouillies dans le sol et en même temps la destruction de ces adventices déjà émergées. D’autre part, nous pouvons noter aussi la diversification des cultures dont les semences peuvent être des voies de dissémination des adventices tout comme l'apport de la fumure organique et l'usage des outils de travail du sol (Sarr et al., 2007).

\section{Conclusion:}

Cette étude a permis de déterminer la structure et les caractéristiques de la flore adventice de la ferme agricole de l'université Gaston Berger de Saint-Louis au Sénégal. Il ressort de cette étude que cette flore est diversifiées avec 149 espèces réparties dans 90 genres appartenant à 30 familles. Les Poaceae $(18,8 \%)$ et les Fabaceae $(15,4 \%)$ sont les familles les plus représentées dans la flore. Cette flore est principalement constituée de thérophytes $(79,9 \%)$ et de chaméphytes $(10,7 \%)$. Elle présente une diversité biogéographique composée pour l'essentiel, de $29,5 \%$ d'espèces pantropicales et de $28,2 \%$ d'espèces africaines qui totalisent plus de la moitié $(57,7 \%)$ des espèces répertoriées de la flore. L'étude a montré que les espèces rares sont dominantes avec 52\%. Ces informations sont utiles pour une maîtrise de la concurrence des adventices vis à vis aux cultures dans l'exploitation agricole de la ferme. En effet, la connaissance de ces adventices qui composent cette flore est primordiale pour l'élaboration de moyens de contrôle performant.

\section{References:}

1. Akobundu I. O., \& Agyakwa C. W. (1989). Guides des adventices d'Afrique de 1'Ouest. 522p.

2. APG III. (2009). An update of the Angiosperm Phylogeny Group classification for the orders and families of flowering plants: Botanical Journal of the Linnean Society, 161: 105-121.

3. Bassène C. (2014). La flore adventice dans les cultures de maïs (Zea mays L.) dans le sud du Bassin arachidier : structure, nuisibilité et mise au point d'un itinéraire de désherbage. Thèse Unique. UCAD, $189 \mathrm{p}$.

4. Bassène C., Mbaye M. S., Camara A. A., Kane A., Gueye M., Sylla S. N., Noba K. (2014). Flore des systèmes agropastoraux de la Basse 
Casamance (Sénégal) : cas de la communauté rurale de Mlomp. Int. J. Biol. Chem.Sci. 8(5): (pp. 2258 - 2273). http://dx.doi.org/10.4314/ijbcs.v8i5.28.

5. Bassène C., Mbaye M. S., Kane A., Diangar S., Noba K. (2012). Flore adventice du maïs (Zea mays L.) du Bassin arachidier (Sénégal) : structure et nuisibilité des espèces. Journal of Applied Biosciences. 59: (pp. 4301 - 4320). https://dx.doi.org/10.4314/jab.v118i1.1.

6. Berhaut J. (1967). Flore du Sénégal. $2^{\text {ème }}$ Ed. Dakar, Clairafrique. 485 p.

7. Berhaut J. (1971 - 1991). Flore illustrée du Sénégal. Tome I, II, III, IV, V et VI. Gouvernement du Sénégal, Dakar.

8. Caratini R. (1985). Botanique 4. Bordas, paris, France

9. Diack M. (2011). Rapport annuel d'activités de la ferme agricole de l'Université Gaston Berger. 68p.

10. Diack M., \& Loum M. (2014). Caractérisation par approche géostatistique de la variabilité des propriétés du sol de la ferme agropastorale de l'université Gaston Berger de Saint-Louis, dans le Bas Delta du fleuve Sénégal. Revue de géographie du Laboratoire Leïdi (12) $0851 \quad$ - 2515. http://www.ugb.sn/revues1sh/images/LEIDI/LEIDI12/LEIDI12-01.

11. Drevon, J.J. (2003). Fixation symbiotique de l'azote et développement durable dans le bassin méditerranéen. Edition INRA.

12. Hannachi A. (2010). Etude des mauvaises herbes des cultures de la région de Batna : systématique, biologie et écologie.Mémoire pour l'obtention du diplôme de magister en sciences agronomiques. Univ. Ferhat Abbas-Setif (UFAS) (algerie). Faculté des Sciences département d'agronomie. $124 \mathrm{p}$.

13. ISRA. (2008). Etat des ressources phytogénétiques pour l'alimentation et l'agriculture dans le monde : contribution du Sénégal au second rapport. 57p.

14. Le Bourgeois T. \& Merlier H. (1995). Adventrop : les adventices d'Afrique Souda-Sahélienne. CIRAD-CA. Montpellier. 637p.

15. Le Bourgeois T., Marnotte P. (2002). Modifier les itinéraires techniques : la lutte contre les mauvaises herbes. In : Mémento de l'agronome. Montpellier, France, CIRAD. 684 p.

16. Lebrun J. P. \& Stork A. (1991, 1992, 1995 et 1997). Enumération des plantes à fleurs d'Afrique tropicale. Vol, 1, II, III, IV. Edition des Conservatoires et Jardin botaniques de la ville de Genève.

17. Mbaye M., S. (2013). Association mil [Pennisetum glaucum (L.) R.Br] et niébé [Vigna unguiculata (L.) Walp.] : arrangement spatiotemporel des cultures, structures, dynamique et concurrence de la flore 
adventice et proposition d'un itinéraire technique. Thèse de Doctorat d'état. Université Ckeikh Anta Diop de Dakar. 236 p.

18. Merlier et H., \& Montegut T. (1982). Adventices tropicales. ORSTOM-GERDAT-ENSH.490p.

19. Mugnier J. (2008). Nouvelle flore illustrée du Sénégal et régions voisines.

20. Noba K., Bâ A. T. , Caussanel J-P., Mbaye M. S., Barralis G. (2004). Flore adventice des cultures vivrières dans le sud du Bassin arachidier (Sénégal). Webbia, $\quad 59 \quad$ (2) : 293-308. http://www.tandfonline.com/loi/tweb20.

21. Noba K. (2002). La flore adventice dans le sud du Bassin arachidier (Sénégal): structure, dynamique et impact sur la production du mil et de l'arachide. Thèse de Doctorat d'Etat. UCAD/FST. 137p.

22. Poillecot P. (1995, 1999). Les Poaceae de Côte d'Ivoire. Vol. 50 et 56. Ed. des Conservatoires et Jardin botaniques de la ville de Genève, Boissiera.

23. Rameau J.-C., Mansion D., Dumé G., Gauberville C.,Bardat J., Bruno E. et Keller R. (2008). Flore forestière française guide illustré 3 Régions méditerranéenne. 2419p.

24. Raunkier C. (1934). The life froms of plants and statistical Plants Geography. Clarendron Press, Oxford. 623p.

25. Sarr R. S., Mbaye M. S. \& Ba T. (2007). La flore adventice des cultures d'oignons dans la zone péri-urbaine de Dakar (Niayes) Sénégal. Webbia, 62 (2). 205-216. http://www.tandfonline.com/loi/tweb20.

26. Schneider A. \& Guyghe C. (2015). Les légumineuses pour des systèmes agricoles et alimentaires durables. Edition Quae. 512 p.

27. Taleb A., Bouhache M. \& Rzozi S.B. (1998). Flore adventice des céréales d'automne au Maroc. Actes Inst. Agron. Vet. Hassan II, 18(2): 121-130.

https://www.agrimaroc.org/index.php/Actes_IAVH2/article/downloa d/32/11.

28. Traoré H., \& Maillet J. (1992). Flore adventice des cultures céréalières annuelles du Burkina Fasso. Weed research, 32:. 279-293. https://agritrop.cirad.fr/263927/1/document_263927.

29. Trochain J. L. (1966). Types Biologiques chez les végétaux intertropicaux (Angiospermes) Bull. Sco. Bot. de France, pp. 188-196.

30. Zidane L., Salhi S., Fadli M., El Antri M., Taleb A., Douira A. (2010). Étude des groupements d'adventices dans le Maroc occidental. Biotechnol. Agron. Soc.Environ.14(1): 153-166. http://www.pressesagro.be/base/text/v14n1/153. 